\title{
Testing normality of chosen R-estimates used in deformation analysis
}

DOI: https://doi.org/10.1515/jogs-2020-0002

Received December 6, 2019; accepted March 3, 2020

\begin{abstract}
The normal distribution is one of the most important distribution in statistics. In the context of geodetic observation analyses, such importance follows Hagen's hypothesis of elementary errors; however, some papers point to some leptokurtic tendencies in geodetic observation sets. In the case of linear estimators, the normality is guaranteed by normality of the independent observations. The situation is more complex if estimates and/or the functional model are not linear. Then the normality of such estimates can be tested theoretically or empirically by applying one of goodness-of-fit tests.

This paper focuses on testing normality of selected variants of the Hodges-Lehmann estimators (HLE). Under some general assumptions the simplest HLEs have asymptotical normality. However, this does not apply to the Hodges-Lehmann weighted estimators (HLWE), which are more applicable in deformation analysis. Thus, the paper presents tests for normality of HLEs and HLWEs. The analyses, which are based on Monte Carlo method and the Jarque-Bera test, prove normality of HLEs. HLWEs do not follow the normal distribution when the functional model is not linear, and the accuracy of observation is relatively low. However, this fact seems not important from the practical point of view.
\end{abstract}

Keywords: Displacement analysis, Hodges-Lehmann estimates, Jarque-Bera test, Normality, R-estimation

\section{Introduction}

The normal distribution is one of the most important distribution in theory of probability, statistics, estimation and hence the adjustment. This is due to the fact that it has well-known mathematic properties and many stochastic phenomena or processes can be described by applying

\footnotetext{
*Corresponding Author: R. Duchnowski: Department of Geodesy, University of Warmia and Mazury in Olsztyn, Olsztyn 10-719, Poland, E-mail: robert.duchnowski@uwm.edu.pl

P. Wyszkowska: Department of Geodesy, University of Warmia and Mazury in Olsztyn, Olsztyn 10-719, Poland
}

the normal distribution (e.g., Wiśniewski, 2014; Lehmann, 2015). In the context of analysis, processing or adjustment of geodetic observations, the importance of such a distribution follows Hagen's hypothesis of elementary errors which leads to the distribution in question. It is usually assumed that geodetic observation errors are normally distributed; however, some analyses point at slight leptokurtosis of some types of the observations (see, for example, Wiśniewski, 2014; Lehmann, 2015; Duchnowski and Wyszkowska, 2017). Such an assumption is also important in the adjustment processes when we need to estimate the distribution of the adjusted parameters, or more generally, distributions of the estimators. In the case of linear estimators, for example estimators of the least squares method, the normality of the estimates is guaranteed by normality of the independent observations (due to the properties of the normal distribution). On the other hand, many practical problems of geodesy or surveying require application of other estimation methods which might be nonlinear. Here, we should pay special attention to robust methods. Several robust estimation methods (e.g., M-estimation, Restimation, or in some sense $\mathrm{M}_{\text {split }}$ estimation) are often applied in geodetic computation to deal with outlying observations of different origins (e.g., Yang et al., 2002; Xu, 2005; Duchnowski, 2013; Nowel and Kamiński, 2014; Wiśniewski, et. al. 2019). Robust estimation methods are supposed to cope with outliers by design, unless the number of outliers does exceed the method breakdown point (e.g., $\mathrm{Xu}, 2005)$. An alternative for robust estimation is data snooping in different variants which are dedicated to particular problems of geodetic data analysis (e.g., Lehmann, 2012, 2015; Prószyński, 2015; Rofatto et al., 2018; Zaminpardaz and Teunissen, 2019). Data snooping involves statistical tests (global and local) to identifying outliers. Then one can reject the suspected observations from the observation set and conduct the estimation in a classical way. Note that the critical values which are applied in the statistical tests in question result from the assumed stochastic models, hence from assumed distribution of observations and estimated parameters (Lehmann, 2015; Prószyński, 2015; Nowel, 2016). 
This paper concerns R-estimates which can be applied in deformation analysis and which are all nonlinear. Considering robust estimation or data snooping, it is advisable to have knowledge about distribution of such estimates. The normality of R-estimates has been discussed since the pioneer paper of Hodges and Lehmann (1963). It was proved that generally R-estimates, including HodgesLehmann estimates (HLEs), have asymptotical normality (Hettmansperger and McKean, 2011). However, that conclusion does not apply to the Hodges-Lehmann weighted estimators (HLWE), which are more applicable in geodetic computations (Duchnowski, 2013). The normality of such estimates can be investigated from the theoretical point of view as well as in an empirical way. In the latter case we can apply one of the well-known goodness-of-fit tests. Thus, the paper focuses on normality of R-estimates and presents tests for normality of HLEs and HLWEs for the finite samples (for different small number of observations). The analyses are based on Crude Monte Carlo method and the Jarque-Bera test (JB), and more precisely on testing the distribution of JB statistic which should asymptotically follow the $\chi^{2}$ distribution.

\section{R-estimates}

The basic R-estimates, namely the estimates that are based on rank tests, were introduced by Hodges and Lehmann (1963) which consider one sample problem as well as two sample problem. From the geodetic point of view, the second problem is much more important since such R-estimates are natural estimates of the shift between two samples, hence, under some reasonable assumptions, they are also the estimates of the point displacements (Duchnowski, 2013). Thus, let us assume that independent samples $x_{1}, x_{2}, \ldots, x_{m}$ and $y_{1}, y_{2}, \ldots, y_{k}$ are the sets of realizations of random variables, respectively $X_{j}$ and $Y_{i}$. Let the variables $X_{j}$ (or $Y_{i}$ ) have the common expected value and variance. When one applies the Wilcoxon test and respective test statistic, then HLE of the shift between such samples, denoted as $\hat{\Delta}^{H L}$, can be computed by applying the following formula (Hodges and Lehmann, 1963)

$$
\hat{\Delta}^{H L}=\operatorname{med}\left(y_{i}-x_{j}\right)
$$

where: med - a median operator. Such a formula is wellknown and successful in many statistical problems. In the case of surveying computations, the general assumptions of HLE are not always fulfilled. Most of all, accuracy of observations might differ from one another. Thus, Duchnowski (2013) proposed to change the assumptions in question in order to accept different observation variances. This leads to the other variant of HLE, namely the Hodges-Lehmann weighted estimates (HLWE) in the form

$$
\hat{\Delta}^{H L W}=\operatorname{med} w\left(y_{i}-x_{j}\right)
$$

where: medw - a weighted median operator. Both formulas seem very similar, but they differ much from each other. First, they stem from application of different test statistics, and the computation of the weighted median is also more complicated, and it can be done by application of a partial heapsort method (for the detailed description of the method, see Duchnowski, 2013).

\section{Testing Normality of R-Estimates}

As it was mentioned, normality of the chosen R-estimates can be tested in an empirical way. Here, we apply the Jarque-Bera test for normality. Of course, one can choose other test which can be more powerful (for example, Thadewald and Büning, 2007); however, JB test is very simple in use and for the purpose of this paper application of this test seems enough. JB test is based on the following test statistic (Jarque and Bera, 1980; Wiśniewski, 2017)

$$
J B=\frac{n}{6}\left[\beta_{1}+\frac{\left(\beta_{2}-3\right)^{2}}{4}\right]
$$

where: $\beta_{1}-$ Pearson's sample squared skewness and $\beta_{2}$ - the sample kurtosis; $n$ - a number of observations. If $J B<\chi_{\alpha}^{2}$, then the null hypothesis $H_{0}: Z \sim N\left(0, \sigma^{2}\right)$ should not be rejected ( $\sigma^{2}-$ the variance and $\chi_{\alpha}^{2}$ - the critical value for a given significance level $\alpha$ ). The statistic $J B$ is asymptotically $\chi^{2}$ distributed with two degrees of freedom if the null hypothesis is true. Table 1 presents the critical values for the chosen significance level.

Here, normality of the chosen R-estimates is tested by applying simulated observation sets. Thus, let $x_{1}, x_{2}, \ldots, x_{m}$ and $y_{1}, y_{2}, \ldots, y_{k}$ be simulated from respective normal distributions, and the shift between the samples be estimated by applying R-estimates of Eqs. (1 or 2). By repeating such computation for $n$ times, we can obtain the set of the estimates for which we can conduct $J B$ test. For the assumed significance level, we can decide about rejecting or not the null hypothesis. Note that in such a case the probability of rejecting the "true" null hypothesis is in fact equal to $\alpha$. For that reason and since we are simulating the observation sets, we decided to conduct the analysis of normality of R-estimates in another way. Thus, we repeat the whole procedure which is described here for $l$ times and hence obtain the set of 
Table 1. Critical values in $J B$ test

\begin{tabular}{cccccccc}
\hline$\alpha$ & 0.010 & 0.050 & 0.250 & 0.500 & 0.750 & 0.950 & 0.990 \\
\hline$X_{\alpha}^{2}$ & 9.210 & 5.992 & 2.773 & 1.386 & 0.573 & 0.103 & 0.020 \\
\hline
\end{tabular}

the values of $J B$ statistic. Now, we can check if such a set is $\chi^{2}$ distributed. For example, one can compare the values of the empirical cumulative distribution function (ECDF) to the theoretical values of $\mathrm{CDF}$ at the critical values. Another approach is to compare empirical significance levels, $\alpha^{e m p}$, to the assumed values of $\alpha$. In fact, these two approaches are equivalent.

Let us now test normality of the estimates of Eqs. (1 and 2). For all simulations we assume that $k=m$ (the observation sets are the same size), $n=2000$ and $l=1000$, which seems enough.

\subsection{Hodges-Lehmann Estimates}

First, let us investigate the normality of the basic HodgesLehmann estimates for different number of observations $k$. Now, let $\sigma^{2}=1$. The results based on the simulations are presented in Table 2.

The empirical values $\alpha^{e m p}$ are close to the theoretical ones; however, the differences are not negligible (maximal 0.020). To find out if such differences are significant or not, one can refer to Kolmogorov-Smirnov goodness-of-fit test, K-S test (for example, Thadewald and Büning, 2007). K-S statistic is equal to the maximum absolute difference between ECDF and the underlying CDF. For $\alpha=0.050$ and $l=1000$, the critical value of K-S test is equal to 0.043 (O'Connor and Kleyner, 2012). Thus, the absolute differences which are obtained here are not statistically significant.

\subsection{Hodges-Lehmann Weighted Estimates}

In the case of HLWEs, the observations might differ from one another in accuracy. Thus, in the following simulation procedures we might assume different a priori accuracies of observations. In order to simplify notation, the observation standard deviations are included into the respective vectors $\sigma_{X}$ and $\sigma_{Y}$.

$k=3$

Variant I:

$\sigma_{X}=\sigma_{Y}=\left[\begin{array}{lll}1 & 2 & 4\end{array}\right]^{T}$

Variant II:

$\sigma_{X}=\left[\begin{array}{lll}1 & 1 & 1\end{array}\right]^{T}, \sigma_{Y}=\left[\begin{array}{lll}1 & 4 & 16\end{array}\right]^{T}$ $k=5$

Variant I:

$\sigma_{X}=\sigma_{Y}=\left[\begin{array}{lllll}1 & 2 & 2 & 4 & 4\end{array}\right]^{T}$

Variant II:

$\sigma_{X}=\left[\begin{array}{lllll}1 & 1 & 1 & 1 & 1\end{array}\right]^{T}, \sigma_{Y}=\left[\begin{array}{lllll}1 & 2 & 4 & 8 & 16\end{array}\right]^{T}$

$k=10$

Variant I:

$\sigma_{X}=\sigma_{Y}=\left[\begin{array}{llllllllll}1 & 1 & 2 & 2 & 4 & 4 & 8 & 8 & 16 & 16\end{array}\right]^{T}$

Variant II:

$\sigma_{X}=\left[\begin{array}{llllllllll}1 & 1 & 1 & 1 & 1 & 1 & 1 & 1 & 1 & 1\end{array}\right]^{T}$,

$\sigma_{Y}=\left[\begin{array}{llllllllll}1 & 1 & 2 & 2 & 4 & 4 & 8 & 8 & 16 & 16\end{array}\right]^{T}$

The empirical significance levels for several variants are presented in Table 3. The maximal absolute differences are: 0.022 for $k=3,0.028$ for $k=5$ and 0.040 for $k=10$ (mean absolute differences are 0.009, 0.012 and 0.012, respectively). Thus, referring to K-S test, the differences are not statistically significant. One can say that the results confirm normality of HLWEs.

\subsection{Hodges-Lehmann Weighted Estimates in deformation analysis}

The last example concerns the practical application of R-estimates. Let us consider the example horizontal network established for deformation analysis which was introduced in (Duchnowski, 2013). Here, we consider only the objective point 1 , thus the network consists of three reference points and one object point; observations including distances $(D)$ and angles $(A)$ are measured at two epochs. It is assumed that $\sigma_{A}=0.0005^{g}, \sigma_{D}=0.002 \mathrm{~m}$, and the simulated displacements $\Delta_{X 1}=0.040 \mathrm{~m}$ and $\Delta_{Y 1}=0.020 \mathrm{~m}$. The network structure, the coordinates of the reference points as well as the theoretical observations are presented in Fig. 1 (for the detailed description of the network, see Duchnowski, 2013). The application of HLWE in displacement analysis is based on computation of the coordinates of the point in all possible independent ways (by applying the measurements and different kinds of intersections or resection). One can also assess the accuracy of such computed coordinates from the accuracies of the measurements $\sigma_{A}, \sigma_{D}$ and the law of variance propagation. Such a procedure should be repeated for both measurement epochs and both point coordinates. Then, the es- 
Table 2. Empirical significance levels $\alpha^{e m p}$ for HLEs

\begin{tabular}{ccccc}
\hline$\alpha$ & \multicolumn{3}{c}{$\alpha^{e m p}$} \\
\cline { 2 - 5 } & $k=3$ & $k=5$ & $k=10$ & $k=20$ \\
\hline 0.010 & 0.014 & 0.017 & 0.015 & 0.020 \\
0.050 & 0.052 & 0.059 & 0.043 & 0.064 \\
0.250 & 0.245 & 0.260 & 0.234 & 0.246 \\
0.500 & 0.480 & 0.503 & 0.487 & 0.491 \\
0.750 & 0.758 & 0.736 & 0.754 & 0.737 \\
0.950 & 0.958 & 0.956 & 0.953 & 0.944 \\
0.990 & 0.990 & 0.991 & 0.989 & 0.986 \\
\hline
\end{tabular}

Table 3. Empirical significance levels $\alpha^{e m p}$ for HLWES

\begin{tabular}{ccccccc}
\hline \multirow{2}{*}{$\alpha$} & \multicolumn{7}{c}{$\alpha^{e m p}$} \\
\cline { 2 - 7 } & \multicolumn{3}{c}{$k=3$} & \multicolumn{3}{c}{$k=5$} \\
\hline 0.010 & Variant I & Variant II & Variant I & Variant II & Variant I & Variant II \\
\hline 0.050 & 0.020 & 0.010 & 0.026 & 0.014 & 0.012 & 0.020 \\
0.250 & 0.062 & 0.040 & 0.075 & 0.057 & 0.060 & 0.062 \\
0.500 & 0.255 & 0.231 & 0.270 & 0.246 & 0.250 & 0.270 \\
0.750 & 0.487 & 0.486 & 0.528 & 0.489 & 0.512 & 0.496 \\
0.950 & 0.754 & 0.728 & 0.772 & 0.737 & 0.790 & 0.772 \\
0.990 & 0.954 & 0.947 & 0.958 & 0.944 & 0.958 & 0.958 \\
\hline
\end{tabular}

timator of Eq. (2) can be used to obtain the estimates $\hat{\Delta}_{X 1}^{H L W}$ and $\hat{\Delta}_{Y 1}^{H L W}$, respectively. Let us now test the normality of such estimates in the way which is very similar to the previous tests. Thus, let first $\sigma_{A}=0.0005^{g}, \sigma_{D}=0.002 \mathrm{~m}$, as it was in the paper mentioned. In the other variants considered the standard deviations given might be 10, 100, 1000 or 10000 times bigger.

Variant I:

$\sigma_{A}=0.0005^{g}, \sigma_{D}=0.002 \mathrm{~m}$

Variant II:

$\sigma_{A}=0.005^{g}, \sigma_{D}=0.02 \mathrm{~m}$

Variant III:

$\sigma_{A}=0.5^{g}, \sigma_{D}=2 \mathrm{~m}$

Variant IV:

$\sigma_{A}=5^{g}, \sigma_{D}=20 \mathrm{~m}$

Variant V:

$\sigma_{A}=0.0005^{g}, \sigma_{D}=0.02 \mathrm{~m}$

Variant VI:

$\sigma_{A}=0.0005^{g}, \sigma_{D}=0.2 \mathrm{~m}$

Variant VII:

$\sigma_{A}=0.0005^{g}, \sigma_{D}=2 \mathrm{~m}$

Variant VIII:

$\sigma_{A}=0.0005^{g}, \sigma_{D}=20 \mathrm{~m}$

Variant IX:

$\sigma_{A}=0.005^{g}, \sigma_{D}=0.002 \mathrm{~m}$

Variant $\mathrm{X}$ :

$\sigma_{A}=0.05^{g}, \sigma_{D}=0.002 \mathrm{~m}$

Variant XI:
$\sigma_{A}=0.5^{g}, \sigma_{D}=0.002 \mathrm{~m}$

Variant XII:

$\sigma_{A}=5^{g}, \sigma_{D}=0.002 \mathrm{~m}$

The respective empirical significance levels are presented in Table 4. In the first three variants, the differences between the theoretical and empirical $\alpha$ are relatively small (maximal 0.016), hence they are not statistically significant. In Variant IV, some differences are bigger than the critical value 0.043 as for the coordinate $Y 1$ ( $\operatorname{such} \alpha_{Y 1}^{e m p}$ are underlined in Table 4), thus, the null hypothesis should be rejected. Since the null hypothesis is not rejected for all $\hat{\Delta}_{X 1}^{H L W}$, the lack of normality of $\hat{\Delta}_{Y 1}^{H L W}$ might result not only from the low observation accuracy but also from the network structure.

Let us now consider the variants in which accuracies of distances and angles are at the different levels. Variants V-VIII concern the constant high accuracy of angles and the decreasing accuracy of distances. The results, which are presented in Table 5, are relatively close to the theoretical values of the significance level for all the variants. Thus, HLWEs seem to follow normal distribution.

The next variants, namely Variants IX-XII, assume the constant high accuracy of distances and the decreasing accuracy of angles. The results, see Table 6 , are close to the theoretical values for the first three variants. On the other hand, the empirical significance levels obtained in Variant XII are often far from such values for the both coordinates considered. The values $\alpha_{X 1}^{e m p}$ and $\alpha_{Y 1}^{e m p}$, for 




Fig. 1. Simulated horizontal network
Coordinates of reference points:

$X_{101}=100.00 \mathrm{~m}, Y_{101}=100.00 \mathrm{~m}$

$X_{102}=50.00 \mathrm{~m}, Y_{102}=200.00 \mathrm{~m}$

$X_{103}=110.00 \mathrm{~m}, Y_{103}=250.00 \mathrm{~m}$

Distances in epoch I: $\quad$ Distances in epoch II:

$D_{101-1}=174.9286 \mathrm{~m} \quad D_{101-1}=174.9732 \mathrm{~m}$

$D_{102-1}=200.2498 \mathrm{~m} \quad D_{102-1}=200.2888 \mathrm{~m}$

$D_{103-1}=152.3155 \mathrm{~m} \quad D_{103-1}=152.3444 \mathrm{~m}$

Angles in epoch I: $\quad$ Angles in epoch II:

$A_{1-101-102}=95.1126^{\mathrm{g}} \quad A_{1-101-102}=95.1138^{\mathrm{g}}$

$A_{1-101-103}=61.3580^{\mathrm{g}} \quad A_{1-101-103}=61.3592^{\mathrm{g}}$

$A_{101-102-1}=67.3028^{\mathrm{g}} \quad A_{101-102-1}=67.3098^{\mathrm{g}}$

$A_{1-102-103}=47.4089^{\mathrm{g}} \quad A_{1-102-103}=47.4019^{\mathrm{g}}$

$A_{102-103-1}=129.9954^{\mathrm{g}} \quad A_{102-103-1}=130.0096^{\mathrm{g}}$

$A_{101-103-1}=78.4617^{\mathrm{g}} \quad A_{101-103-1}=78.4759^{\mathrm{g}}$

$A_{102-1-101}=37.5846^{\mathrm{g}} \quad A_{102-1-101}=37.5764^{\mathrm{g}}$

$A_{103-1-102}=22.5958^{\mathrm{g}} \quad A_{103-1-102}=22.5885^{\mathrm{g}}$

Table 4. Empirical significance levels $\alpha_{X 1}^{e m p}$ and $\alpha_{Y 1}^{e m p}$ for HLWEs in horizontal network

\begin{tabular}{ccccccccc}
\hline \multirow{2}{*}{$\alpha$} & \multicolumn{2}{c}{ Variant I } & \multicolumn{2}{c}{ Variant II } & \multicolumn{2}{c}{ Variant III } & \multicolumn{2}{c}{ Variant IV } \\
\cline { 2 - 8 } & $\alpha_{X 1}^{\text {emp }}$ & $\alpha_{Y 1}^{\text {emp }}$ & $\alpha_{X 1}^{\text {emp }}$ & $\alpha_{Y 1}^{\text {emp }}$ & $\alpha_{X 1}^{\text {emp }}$ & $\alpha_{Y 1}^{\text {emp }}$ & $\alpha_{X 1}^{\text {emp }}$ & $\alpha_{Y 1}^{\text {emp }}$ \\
\hline 0.010 & 0.013 & 0.013 & 0.012 & 0.016 & 0.013 & 0.018 & 0.013 & $\underline{0.076}$ \\
0.050 & 0.049 & 0.056 & 0.053 & 0.058 & 0.051 & 0.061 & 0.066 & $\underline{0.160}$ \\
0.250 & 0.240 & 0.235 & 0.235 & 0.234 & 0.240 & 0.235 & 0.276 & $\underline{0.385}$ \\
0.500 & 0.494 & 0.493 & 0.498 & 0.491 & 0.501 & 0.497 & 0.502 & $\underline{0.624}$ \\
0.750 & 0.748 & 0.748 & 0.748 & 0.739 & 0.747 & 0.736 & 0.754 & $\underline{0.797}$ \\
0.950 & 0.934 & 0.962 & 0.960 & 0.949 & 0.949 & 0.942 & 0.953 & 0.964 \\
0.990 & 0.984 & 0.993 & 0.990 & 0.988 & 0.991 & 0.992 & 0.993 & 0.993 \\
\hline
\end{tabular}

Table 5. Empirical significance levels $\alpha_{X 1}^{e m p}$ and $\alpha_{Y 1}^{e m p}$ for HLWEs in horizontal network

\begin{tabular}{ccccccccc}
\hline \multirow{2}{*}{$\alpha$} & \multicolumn{2}{c}{ Variant V } & \multicolumn{2}{c}{ Variant VI } & \multicolumn{2}{c}{ Variant VII } & \multicolumn{2}{c}{ Variant VIII } \\
\cline { 2 - 8 } & $\alpha_{X 1}^{\text {emp }}$ & $\alpha_{Y 1}^{\text {emp }}$ & $\alpha_{X 1}^{\text {emp }}$ & $\alpha_{Y 1}^{\text {emp }}$ & $\alpha_{X 1}^{\text {emp }}$ & $\alpha_{Y 1}^{e m p}$ & $\alpha_{X 1}^{e m p}$ & $\alpha_{Y 1}^{\text {emp }}$ \\
\hline 0.010 & 0.013 & 0.023 & 0.009 & 0.015 & 0.007 & 0.012 & 0.012 & 0.015 \\
0.050 & 0.057 & 0.065 & 0.052 & 0.057 & 0.048 & 0.047 & 0.055 & 0.052 \\
0.250 & 0.257 & 0.257 & 0.242 & 0.262 & 0.233 & 0.230 & 0.249 & 0.237 \\
0.500 & 0.511 & 0.507 & 0.497 & 0.503 & 0.535 & 0.482 & 0.499 & 0.504 \\
0.750 & 0.757 & 0.742 & 0.758 & 0.759 & 0.770 & 0.755 & 0.738 & 0.758 \\
0.950 & 0.954 & 0.953 & 0.957 & 0.948 & 0.957 & 0.953 & 0.953 & 0.957 \\
0.990 & 0.988 & 0.988 & 0.992 & 0.986 & 0.991 & 0.991 & 0.990 & 0.990 \\
\hline
\end{tabular}

which the differences exceed the critical value 0.043 , are underlined in Table 6 . What is more, the worse results (the bigger differences between theoretical and empirical values) are acquired for coordinate $X 1$, contrary to Variant IV. This results from the propagation of the expected values from angles and distances to coordinates for nonlinear functions (intersections and resection) and when accuracies of measurements are much different, see for example (Wyszkowska, 2017).
The theoretical and empirical significance levels which are obtained in Variants IV and XII are presented in Fig. 2. 
Table 6. Empirical significance levels $\alpha_{X 1}^{e m p}$ and $\alpha_{Y 1}^{e m p}$ for HLWEs in horizontal network

\begin{tabular}{ccccccccc}
\hline \multirow{2}{*}{$\alpha$} & \multicolumn{2}{c}{ Variant IX } & \multicolumn{2}{c}{ Variant X } & \multicolumn{2}{c}{ Variant XI } & \multicolumn{2}{c}{ Variant XII } \\
\cline { 2 - 8 } & $\alpha_{X 1}^{\text {emp }}$ & $\alpha_{Y 1}^{\text {emp }}$ & $\alpha_{X 1}^{\text {emp }}$ & $\alpha_{Y 1}^{\text {emp }}$ & $\alpha_{X 1}^{\text {emp }}$ & $\alpha_{Y 1}^{e m p}$ & & $\alpha_{Y 1}^{\text {emp }}$ \\
\hline 0.010 & 0.018 & 0.013 & 0.021 & 0.009 & 0.016 & 0.008 & $\frac{1}{1}$ & 0.048 \\
0.050 & 0.060 & 0.046 & 0.080 & 0.040 & 0.063 & 0.038 & $\frac{1}{1}$ & $\underline{0.109}$ \\
0.250 & 0.242 & 0.240 & 0.288 & 0.225 & 0.249 & 0.223 & $\frac{1}{1}$ & $\underline{0.546}$ \\
0.500 & 0.503 & 0.481 & 0.529 & 0.492 & 0.497 & 0.492 & $\frac{1}{1}$ & 0.788 \\
0.750 & 0.762 & 0.729 & 0.774 & 0.757 & 0.753 & 0.763 & $\underline{1}$ & 0.960 \\
0.950 & 0.950 & 0.945 & 0.952 & 0.951 & 0.949 & 0.954 & $\underline{1}$ & 0.990 \\
0.990 & 0.992 & 0.990 & 0.985 & 0.991 & 0.989 & 0.989 & 1 & \\
\hline
\end{tabular}
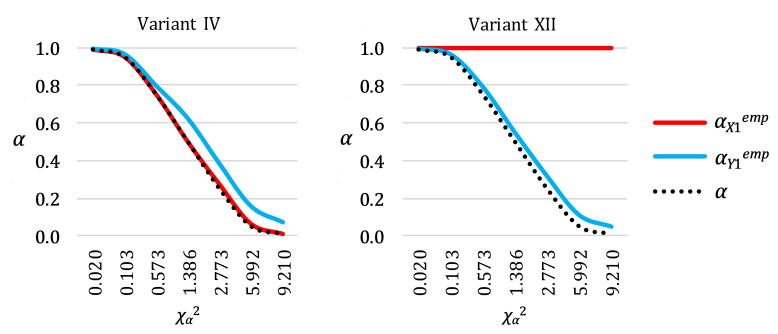

Fig. 2. Empirical significance levels for HLWEs in horizontal network

\section{Conclusions}

The paper presents the tests for normality of the chosen R-estimates which can be applied in geodetic or surveying problems. First, the tests confirm the normality of the basic HLEs of the shift (two sample problem) for the finite samples. The most important and novel part of the paper concerns HLWEs which are more important from the practical point of view. Note that the normality of such estimates has never been tested yet. The simulations show that HLWEs follow the normal distribution in the case of its basic applications. In more complex problem (e.g., deformation analysis) the situation is different. For most of the cases, the normality of HLWEs is also proved. However, HLWEs might not be normally distributed when the accuracy of observations is relatively low. This concerns especially low accuracy of angles. Since geodetic observations usually have much higher accuracy then such a lack of normality seems unimportant from the practical point of view.

Acknowledgements: This work was supported by Department of Geodesy, University of Warmia and Mazury in Olsztyn [statutory research no. 28.610.002-110].

\section{References}

Duchnowski R., 2013, Hodges-Lehmann estimates in deformation analyses, J. Geod., 87, 10, 873-884.

Duchnowski R. and Wyszkowska P., 2017, Leptokurtosis of error distribution and its influence on estimation accuracy. The case of three estimates applied in adjustment of geodetic measurements, In: Kellengerger P. (Ed.), Proceedings of 2017 Baltic Geodetic Congress (BGC Geomatics) (22-25 June 2017, Gdańsk, Poland), IEEE, Piscataway, 237-241.

Hettmansperger T. P. and McKean J. W., 2011, Robust nonparametric statistical methods, Second Edition, CRC Press, Taylor\&Francis Group, Bora Raton.

Hodges Jr. J. L. and Lehmann E. L., 1963, Estimates of location based on rank tests, The Ann. Math. Stat., 34, 2, 598-611.

Jarque C. M., and Bera A. K., 1980, Efficient tests for normality, homoscedasticity and serial independence of regression residuals, Econ. Lett., 6, 3, 255-259.

Lehmann R., 2012, Improved critical values for extreme normalized and studentized residuals in Gauss-Markov models, J. Geod., 86, 1137-1146.

Lehmann R., 2015, Observation error model selection by information criteria vs. normality testing, Stud. Geophys. Geod., 59, 4, 489-504.

Nowel K., 2016, Application of Monte Carlo method to statistical testing in deformation analysis based on robust M-estimation, Surv. Rev., 48, 348, 212-223.

Nowel K. and Kamiński W., 2014, Robust estimation of deformation from observation differences for free control networks, J. Geod., 88, 8, 749-764.

O'Connor P. D. T. and Kleyner A., 2012, Practical reliability engineering, Fifth Edition, John Wiley \& Sons, Ltd.

Prószyński W., 2015, Revisiting Baarda's concept of minimal detectable bias with regard to outlier identifiability, J. Geod., 89, 993-1003.

Rofatto V. F., Matsuoka M. T., Klein I., Veronez M. R., Bonimani M. L. and Lehmann R., 2018, A half-century of Baarda's concept of reliability: a review, new perspectives, and applications, Surv. Rev.

Thadewald T. and Büning H., 2007, Jarque-Bera test and its competitors for testing normality - A power comparison, J. Appl. Stat., 34, 1, 87-105.

Wiśniewski Z., 2014, M-estimation with probabilistic models of geodetic observations, J. Geod., 88, 10, 941-957.

Wiśniewski Z., 2017, $M_{P}$ estimation applied to platykurtic sets of geodetic observations. Geod. Cartography, 66, 1, 117-135. 
Wiśniewski Z., Duchnowski R. and Dumalski A., 2019, Efficacy of $M_{\text {split }}$ estimation in displacement analysis, Sensors, 19, 22, 5047.

Wyszkowska P., 2017, Propagation of uncertainty by Monte Carlo simulations in case of basic geodetic computations, Geod. Cartography, 66, 2, 333-346.

Xu P., 2005, Sign-constrained robust least squares, subjective breakdown point and the effect of weights of observations on robustness, J. Geod., 79, 146-159.
Yang Y., Song L., Xu T., 2002, Robust estimator for correlated observations based on bifactor equivalent weights, J. Geod., 76, 353-358.

Zaminpardaz S. and Teunissen P. J. G., 2019, DIA-datasnooping and identifiability, J. Geod., 93, 1, 85-101. 